Pacific Journal of Mathematics

PECTRAL OPERATORS 


\section{SPECTRAL OPERATORS}

\section{NELSON DUNFORD}

1. Introduction. The present paper and the five following it by S. Kakutani, J. Wermer, W. G. Bade, and J. Schwartz are all related; in them we discuss different aspects of the problem of the complete reduction of an operator. A spectral operator is a linear operator on a complex Banach space which has a resolution of the identity. ${ }^{1}$ It is shown that a bounded operator $T$ is spectral if and only if it has a canonical decomposition of the form

$$
T=S+N
$$

where $S$ is a scalar type operator and $N$ is a generalized nilpotent commuting with $S$. By a scalar type operator is meant a spectral operator $S$ with resolution of the identity $E$ which satisfies the equation

$$
S=\int_{\sigma(S)} \lambda E(d \lambda)
$$

The scalar part $S$ of $T$ and the radical part $N$ of $T$ are uniquely determined by $T$. For analytic functions $f$ one has an operational calculus given by the formula

$$
f(T)=\sum_{n=0}^{\infty} \frac{N^{n}}{n !} \int_{\sigma(T)} f^{(n)}(\lambda) E(d \lambda) .
$$

Some spectral operators are of type $m$; that is, the above formula reduces to

$$
f(T)=\sum_{n=0}^{m} \frac{N^{n}}{n !} \int_{\sigma(T)} f^{(n)}(\lambda) E(d \lambda),
$$

and in Hilbert space conditions on the resolvent are given which are equivalent to the statement that the spectral operator $T$ is of type $m$. Spectral operators $T$

1 Formal definitions will be given later.

Received March 4, 1953. The research contained in this paper was done under Contract onr 609(04) with the Office of Naval Research.

Pacific J. Math. 4 (1954), 321 - 354 
have the property that for every $x$ the analytic function $(\lambda I-T)^{-1} x$ has only single-valued analytic extensions and thus has a maximal extension defined on an open set $\rho(x)$. The spectrum $\sigma(x)$ is defined as the complement of $\rho(x)$. In terms of these concepts it is shown that if $E$ is a resolution of the identity for $T$, then, for closed sets $\sigma$,

$$
E(\sigma) X=[x \mid \sigma(x) \subset \sigma],
$$

This (Theorem 4) is a basic theorem; from it one deduces that the resolution of the identity is unique, as well as the fact that every bounded operator commuting with $T$ commutes with $E(\sigma)$, a fact proved for normal operators on Hilbert space by B. Fuglede [7]. ${ }^{2}$ Let $\mathscr{U}(T, U, \ldots, V)$ be the full $B$-algebra generated by the operators $T, U, \ldots, V$; then we have the following decomposition theorems. If $T$ is spectral and $S$ its scalar part, then, as a vector direct sum,

$$
\mathfrak{U}(T, S)=\mathfrak{U}(S) \oplus R,
$$

where $R$ is the radical in $\mathscr{X}(T, S)$. Furthermore, $\mathscr{X}(S)$ is equivalent to that subalgebra of $C(\sigma(T))$ consisting of uniform limits of rational functions. The algebra $\mathfrak{A}$, which is generated by a spectral operator $T$ and the projections $E(\sigma)$ in its resolution of the identity, is equivalent to

$$
C(\mathbb{M}) \oplus R \text {, }
$$

where $\mathfrak{M}$ is the compact structure space of $\mathscr{U}$ and $R$ is the radical in $\mathscr{X}$. Along these lines we mention the decomposition of the full $B$-algebra $\mathfrak{U}(\tau)$ determined by a family $\tau$ of commuting spectral operators together with their resolutions of the identity. If there is a bounded Boolean algebra of projections in $\mathfrak{X}$ containing all of the projections found among the resolutions of the identity of operators in $\tau$, then

$$
\mathfrak{V}(\tau)=\mathfrak{A} \oplus R,
$$

where $\mathfrak{U}$ is equivalent to the space $C(\mathfrak{R})$ of continuous functions on the space $\mathbb{M}$ of maximal ideals in $\mathfrak{X}(\tau)$ ( or in $\mathscr{U}$ ) and $R$ is the radical in $\mathscr{U}(\tau)$. Furthermore, the adjoint of every operator in $\mathfrak{U}(\tau)$ is a spectral operator. If $\mathfrak{X}$ is reflexive, then every operator in $\mathscr{U}(\tau)$ is a spectral operator. Thus in a reflexive space the sum and product of two commuting spectral operators is a spectral

\footnotetext{
${ }^{2}$ That this conjecture of von Neumann, which was first proved by Fuglede, is a
} corollary of Theorem 4 was pointed out to the author by J. Schwartz. 
operator provided that there is a bounded Boolean algebra containing both resolutions of the identity. W. G. Bade [2] has generalized this by showing that the weakly closed algebra generated by a bounded Boolean algebra of projections in a reflexive space consists entirely of scalar type spectral operators. In this paper Bade has also given sufficient conditions for the strong limit of scalar type spectral operators to be of scalar type. If $X$ is Hilbert space J. Werner [16] has shown that the sum and product of two commuting spectral operators is again a spectral operator. However, S. Kakutani [10] has constructed an example of two commuting operators, each of scalar type, such that their sum is not a spectral operator. W. G. Bade [1] has shown which portions of the theory are valid for unbounded operators and has developed the operational calculus for this case. J. Schwartz [12] has shown that, on a finite interval, the members of a large class of boundary-value problems determine spectral operators. These operators need not be purely differential operators but may also involve difference or integral operators.

2. Notation. By an admissible domain is meant an open set bounded by a finite number of rectifiable Jordan curves. By an admissible contour is meant the boundary of an admissible domain. The class of complex-valued functions analytic and single-valued on some admissible domain containing the spectrum $\sigma(T)$ of the linear operator $T$ is denoted by $F(T)$ or $F(\sigma(T))$. For $f \in F(T)$ the operator $f(T)$ is defined by

$$
f(T)=\frac{1}{2 \pi i} \int_{C} f(\lambda) T(\lambda) d \lambda,
$$

where $C$ is the boundary of some admissible domain containing the spectrum of $T$ upon whose closure $f$ is single-valued and analytic and where $T(\lambda)=(\lambda I-T)^{-1}$ is the resolvent of $T$. The mapping, given by the above formula, of the algebra of analytic functions into an algebra of operators is a homomorphism (See, for example, [3] or [14].) which assigns the operators $I, T$ to the functions $1, \lambda$, respectively. It has the property that $\sigma(f(T))=f(\sigma(T))$. If $f(\lambda)=1$ for $\lambda$ in a component of its domain, and $f(\lambda)=0$ for $\lambda$ in the remaining components, then $f(T)$ is the projection

$$
E(\sigma)=\frac{1}{2 \pi i} \int_{G} T(\lambda) d \lambda,
$$

where $G$ is the boundary of that component upon which $f(\lambda)=1$ and where $\sigma$ is that part of the spectrum $\sigma(T)$ of $T$ bounded by $G$. It is clear that such a 
projection is associated with every subset $\sigma$ of $\sigma(T)$ which is both open and closed in $\sigma(T)$. From the fact that the map $f \longrightarrow f(T)$ is a homomorphism it follows that the map $\sigma \longrightarrow E(\sigma)$ is a homomorphism of the Boolean algebra $B_{0}$ of open and closed sets in $\sigma(T)$ into a Boolean algebra of projection operators. It has the property (see $[2]$ )

$$
\sigma(T, E(\sigma) \mathfrak{X}) \subset \sigma, \sigma \in \mathbb{B}_{0},
$$

where here we have used the notation $\sigma(T, E(\sigma) X)$ for the spectrum of $T$ when considered as an operator in $E(\sigma) X$. Similarly $\rho(T, E(\sigma) \mathfrak{X})$ is the resolvent set of $T$ when considered as an operator in $E(\sigma) X$ and $\rho(T)$ is $\rho(T, X)$. The symbol $B(X)$ will be used for the algebra of all bounded linear transformations in the $B$-space $\mathfrak{X}$.

3. Spectral operators. Let $B$ be a Boolean algebra of subsets of a set $p$. We suppose that $p$ and the void set $\varnothing$ are both in $B$. A homomorphic map $E$ of $B$ into a Boolean algebra of projection operators in the complex $B$-space $\mathfrak{X}$ is called a spectral measure in $\mathfrak{X}$ provided that it is bounded and $E(p)=I$. A spectral measure has then, by definition, the properties

$$
(\alpha)\left\{\begin{array}{cc}
E(\sigma) E(\delta)=E(\sigma \delta), \quad E(\sigma) \cup E(\delta)=E(\sigma \cup \delta), & \sigma, \delta \in \mathbb{B}, \\
E\left(\sigma^{\prime}\right)=I-E(\sigma), \quad E(\varnothing)=0, \quad E(p)=I, & \sigma \in \mathbb{B}, \\
|E(\sigma)| \leq K, & \sigma \in \mathbb{B} .
\end{array}\right.
$$

In the conditions $(\alpha)$ the union of two commuting projection operators is understood to be defined by the equation

$$
A \cup B=A+B-A B \text {. }
$$

This union is a projection whose range is the closed linear manifold determined by the ranges of $A$ and $B$.

An operator $T \in B(X)$ is said to be a spectral operator of class $(B, \Gamma)$ in case

( $\beta$ ) $B$ is a Boolean algebra of sets in the complex plane $p$;

$(\gamma) \Gamma$ is a linear manifold in $\chi^{*}$ which is total; that is, $\Gamma x=0$ only when $x=0$;

$(\delta)$ there is a spectral measure $E$ in $\mathscr{X}$ with domain $B$ such that 


$$
T E(\sigma)=E(\sigma) T, \sigma\left(T, E_{\sigma} X\right) \subset \bar{\sigma}, \quad \sigma \in \mathbb{B}
$$

and

$(\epsilon)$ for every $x \in \mathfrak{X}, x^{*} \in \Gamma$, the function $x^{*} E(\sigma) x$ is countably additive on $B$.

The condition $(\epsilon)$ means that if $\left\{\sigma_{n}\right\}$ is a sequence of disjoint sets in $B$ whose union $\sigma$ is also in $B$ then

$$
\sum_{n} x^{*} E\left(\sigma_{n}\right) x=x^{*} E(\sigma) x, \quad x \in \mathcal{X}, x^{*} \in \Gamma .
$$

In case $B$ is a $\sigma$-field and $\Gamma=\mathfrak{X}^{*}$, the Orlicz-Banach-Pettis theorem (see [11, Theorem 2.32] or [5, p. 322]) shows that the operator-valued set function $E(\sigma)$, $\sigma \in B$, is countably additive on $B$ in the strong operator topology.

An operator $T \in B(X)$ is said to be a spectral operator of class $(\Gamma)$, or simply an operator of class $(\Gamma)$, in case it is a spectral operator of class $(B, \Gamma)$, where $B$ is the set of all Borel sets in the plane. An operator is said to be a spectral operator in case it is a spectral operator of class $(\Gamma)$ for some $\Gamma$ satisfying $(\gamma)$. If $T$ is a spectral operator of type $(\mathcal{B}, \Gamma)$, then any spectral measure in $\chi$ with domain $B$ which satisfies $(\delta)$ and $(\epsilon)$ is called a resolution of the identity for $T$.

THЕОRЕM 1. Let $E$ be a resolution of the identity for the spectral operator T. Then

$$
E(\sigma(T))=I
$$

Proof. Let $\sigma$ be a closed subset of the resolvent set $\rho=\rho(T)$. Then, in view of $(\delta)$, we see that the spectrum of $T$ as an operator in $E_{\sigma} \chi$ is void and hence (see [15]) $E_{\sigma}=0$. Since $\rho$ is a denumerable union of closed sets we have from $(\epsilon)$ that

$$
x^{*} E_{\rho} x=0, \quad x \in \mathfrak{X}, \quad x^{*} \in \Gamma,
$$

and from $(\gamma)$ that $E_{\rho}=0$, and hence $E(\sigma(T))=I$.

For $\lambda \in \rho(T)$ we write, as usual, $T(\lambda)$ for $(\lambda I-T)^{-1}$. In the next theorem we shall show that, for spectral operators, every analytic extension of $T(\lambda) x$ is necessarily single-valued. That this is not the case for an arbitrary operator $T$ is elegantly shown by the following example due to S. Kakutani. 
Consider the space $X$ of functions $f$ analytic in the unit circle $|z| \leq 1$ and for which

$$
f(z)=\sum_{n=0}^{\infty} c_{n} z^{n}, \quad \sum_{n=0}^{\infty}\left|c_{n}\right|^{2}=|f|^{2} .
$$

In this space define $T$ by

$$
T(f, z)=\frac{f(z)-f(0)}{z} .
$$

The spectrum of $T$ is the set of $z$ with $|z| \leq 1$, and for $\lambda \in \rho(T)$ the function $T(\lambda)(g, z)$ may be calculated by solving the equation

$$
(\lambda I-T) f=g
$$

for $f(z)$. An elementary calculation gives

$$
f(z)=\frac{z g(z)-f(0)}{\lambda z-1} .
$$

Since $f(z)$ is analytic when $z=\lambda^{-1}$ we must have

$$
f(0)=\lambda^{-1} g\left(\lambda^{-1}\right)
$$

so that

$$
T(\lambda)(g, z)=\frac{z g(z)-\lambda^{-1} g\left(\lambda^{-1}\right)}{\lambda\left(z-\lambda^{-1}\right)} .
$$

Thus the vector-valued analytic function $T(\lambda) g, \lambda \in \rho(T)$, will have multiplevalued extensions if the function $g$ has a multiple-valued analytic continuation outside the unit circle.

In order to describe the situation discussed in the next theorem certain concepts are introduced. By an analytic extension of $T(\xi) x$ will be meant a function $f$ defined and analytic on an open set $D(f) \supset \rho(T)$ and such that

$$
(\xi I-T) f(\xi)=x
$$

for every $\xi$ in $D(f)$. It is clear that, for such an extension,

$$
f(\xi)=T(\xi) x
$$


for $\xi$ in $\rho(T)$. The function $T(\xi) x$ is said to have the single-valued extension property provided that for every pair $f_{2} g$ of analytic extensions of $T(\xi) x$ we have $f(\xi)=g(\xi)$ for every $\xi$ in $D(f) D(g)$. The union of the sets $D(f)$ as $f$ varies over all analytic extensions of $T(\xi) x$ is called the resolvent set of $x$ and is denoted by $\rho(x)$. The spectrum $\sigma(x)$ of $x$ is defined to be the complement of $\rho(x)$. It is clear that if $T(\xi) x$ has the single-valued extension property then there is a maximal extension $x(\cdot)$ whose domain is $\rho(x)$. In this case $x(\xi)$ is a single-valued analytic function with domain $\rho(x)$ and with $x(\xi)=$ $T(\xi) x, \xi \in \rho(T)$.

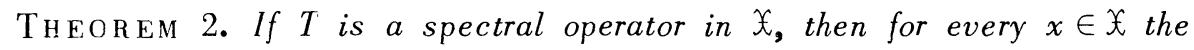
function $T(\xi) x$ has the single-valued extension property.

Proof. Let $f, g$ be two extensions of $T(\xi) x$ and define

$$
h(\xi)=f(\xi)-g(\xi), \quad \xi \in D(f) D(g)
$$

We suppose, in order to make an indirect proof, that for some $\xi_{0} \in D(f) D(g)$ we have $h\left(\xi_{0}\right) \neq 0$. Thus there is a neighborhood $N\left(\xi_{0}\right)$ of $\xi_{0}$ with $N\left(\xi_{0}\right) \subset$ $D(f) D(g)$ and

$$
h(\xi) \neq 0, \quad(\xi I-T) h(\xi)=0, \quad \xi \in N\left(\xi_{0}\right)
$$

The desired contradiction may be obtained from these equations and the following lemma.

I. EMMA 1. Let $E$ be a resolution of the identity for the spectral operator $T$. Let $\sigma$ be a closed set of complex numbers with $\xi_{0} \notin \sigma$. If $\left(\xi_{0} I-T\right) x_{0}=0$ then

$$
E(\sigma) x_{0}=0, E\left(\left\{\xi_{0}\right\}\right) x_{0}=x_{0},
$$

where $\left\{\xi_{0}\right\}$ is the set consisting of the single point $\xi_{0}$.

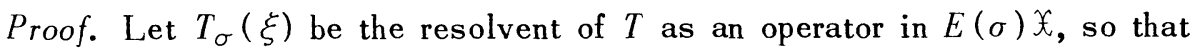

$$
T_{\sigma}\left(\xi_{0}\right)\left(\xi_{0} l-T\right) E(\sigma)=E(\sigma)
$$

But since

$$
\left(\xi_{0} I-T\right) E(\sigma) x_{0}=E(\sigma)\left(\xi_{0} I-T\right) x_{0}=0
$$

we have $E(\sigma) x_{0}=0$. Now let 


$$
\sigma_{n}=\left[\xi|| \xi-\xi_{0} \mid \geq 1 / n\right]
$$

so that $E\left(\sigma_{n}\right) x_{0}=0$; by $(\delta),(\epsilon)$, therefore,

$$
x^{*}\left(I-E\left(\left\{\xi_{0}\right\}\right)\right) x_{0}=\lim _{n} x^{*} L\left(\sigma_{n}\right) x_{0}=0, \quad\left(x^{*} \in \Gamma\right) .
$$

Condition $(\gamma)$ thus shows that $E\left(\left\{\xi_{0}\right\}\right) x_{0}=x_{0}$, and the lemma is proved.

Returning now to the proof of Theorem 2, let

$$
\xi_{n} \neq \xi_{0}, \quad \xi_{n} \in N\left(\xi_{0}\right), \xi_{n} \rightarrow \xi_{0}
$$

Then $h\left(\xi_{n}\right) \rightarrow h\left(\xi_{0}\right)$, and the lemma together with (i) gives

$$
0=E\left(\left\{\xi_{0}\right\}\right) h\left(\xi_{n}\right) \rightarrow E\left(\left\{\xi_{0}\right\}\right) h\left(\xi_{0}\right)=h\left(\xi_{0}\right)
$$

which is a contradiction to (i) and proves the theorem.

THEOREM 3. If $T$ is a spectral operator, the spectrum $\sigma(x)$ is void if and only if $x=0$.

Proof. Using Theorem 2, we see that if $\sigma(x)$ is void then $x(\xi)$ is everywhere defined, single-valued, and hence entire. Since, as $\xi \longrightarrow \propto$, we have

$$
x^{*} x(\xi)=x^{*} T(\xi) x \rightarrow 0
$$

we see that $x^{*} x(\xi)=0$ for all $\xi$. Hence

$$
x^{*} x=x^{*}(\xi I-T) x(\xi)=0,
$$

and $x=0$.

THEOREM 4. Let $T$ be a spectral operator with resolution of the identity $E$, and let $\sigma$ be a closed set of complex numbers. Then

$$
E(\sigma) \mathfrak{X}=[x \mid \sigma(x) \subset \sigma]
$$

Proof. Let $E(\sigma) x=x$, and let $T_{\sigma}(\xi)$ be the resolvent of $T$ as an operator in $E(\sigma) \mathcal{X}$. Then $(\delta)$ shows that

$$
T_{\sigma}(\xi) E_{\sigma} x=T_{\sigma}(\xi) \rightsquigarrow
$$


is an analytic extension of $T(\xi) x$ to $\sigma^{\prime}$, the complement of $\sigma$. Thus $\rho(x) \supset \sigma^{\prime}$, $\sigma(x) \subset \sigma$. Conversely, assume that $\sigma(x) \subset \sigma$ and let $\sigma_{1}$ be a closed subset of the complement $\sigma^{\prime}$ of $\sigma$. Then $T_{\sigma_{1}}(\xi) E\left(\sigma_{1}\right) x$ is an extension of $T(\xi) E\left(\sigma_{1}\right) x$ to $\sigma_{1}^{\prime}$. Also $E\left(\sigma_{1}\right) x(\xi)$ is an extension of $T(\xi) E\left(\sigma_{1}\right) x$ to $\rho(x)$. Thus, from Theorem 2 , it is seen that

$$
E\left(\sigma_{1}\right) x(\xi)=T_{\sigma_{1}}(\xi) E\left(\sigma_{1}\right) x, \xi \in \rho(x) \sigma_{1}^{\prime} .
$$

Since $\sigma, \sigma_{1}$ are disjoint compact sets, there is an admissible contour $C_{1}$ with $\sigma_{1}$ inside $C_{1}$ and $\sigma$ outside. Now let $C$ be a large circle surrounding $\sigma(T)$ so that, since $x(\xi)$ is analytic and single-valued on and within $C_{1}$, we have

$$
\begin{aligned}
E\left(\sigma_{1}\right) x & =\frac{1}{2 \pi i} \int_{C} T(\xi) E\left(\sigma_{1}\right) x d \xi=\frac{1}{2 \pi i} \int_{C} T_{\sigma_{1}}(\xi) E\left(\sigma_{1}\right) x d \xi \\
& =\frac{1}{2 \pi i} \int_{C_{1}} T_{\sigma_{1}}(\xi) E\left(\sigma_{1}\right) x d \xi=\frac{1}{2 \pi i} \int_{C_{1}} E\left(\sigma_{1}\right) x(\xi) d \xi=0 .
\end{aligned}
$$

Let $\sigma_{n}$ be an increasing sequence of closed sets whose union is $\sigma^{\prime}$. Then

$$
x^{*} E\left(\sigma^{\circ}\right) x=\lim _{n} x^{*} E\left(\sigma_{n}\right) x=0, \quad\left(x^{*} \in \Gamma\right),
$$

and so $(\alpha),(\gamma)$ show that $E\left(\sigma^{\prime}\right) x=0, E(\sigma) x=x$.

THEOREM 5. Let $T$ be a spectral operator and $A$ a bounded linear transformation which commutes with $T$. Then A commutes with every resolution of the identity for $T$.

Proof. Let $\sigma, \sigma_{1}$ be disjoint closed sets of complex numbers and let $E$ be a resolution of the identity for $T$. Since

$$
A T(\xi) x=T(\xi) A x,
$$

we see that

$$
\rho(A x) \supset \rho(x), \quad \sigma(A x) \subset \sigma(x) .
$$

Thus Theorem 4 shows that

$$
E(\sigma) A E(\sigma)=A E(\sigma), \quad E(\sigma) A E\left(\sigma_{1}\right)=E(\sigma) E\left(\sigma_{1}\right) A E\left(\sigma_{1}\right)=0 .
$$


Statements $(\gamma),(\epsilon)$ show then that $E(\sigma) A E\left(\sigma^{\prime}\right)=0$, and hence

$$
E(\sigma) A=E(\sigma) A\left[E(\sigma)+E\left(\sigma^{\prime}\right)\right]=E(\sigma) A E(\sigma)+E(\sigma) A E\left(\sigma^{\prime}\right)=A E(\sigma) .
$$

THEOREM 6. If $T$ is a spectral operator, its resolution of the identity is unique.

Proof. If $E, A$ are both resolutions of the identity for $T$, and $\sigma$ is a closed set of complex numbers, then Theorem 4 gives

$$
A(\sigma) E(\sigma)=E(\sigma), E(\sigma) A(\sigma)=A(\sigma),
$$

and $(\delta)$ together with Theorem 5 gives

$$
A(\sigma) E(\sigma)=E(\sigma) A(\sigma) .
$$

Thus for closed sets $\sigma, A(\sigma)=E(\sigma)$, and $(\gamma),(\epsilon)$ show that this same equality holds for every Borel set $\sigma$.

THEOREM 7. Let E be a spectral measure whose domain consists of the Borel sets in the plane and which vanishes on the complement of the compact set $\sigma$. Then, for every scalar function $f$ continuous on $\sigma$, the Riemann integral $\int_{\sigma} f(\lambda) E(d \lambda)$ exists in the uniform operator topology, and

$$
\left|\int_{\sigma} f(\lambda) E(d \lambda)\right| \leq \sup _{\lambda}|f(\lambda)| v(E)
$$

where $v(E)$ is a constant depending only upon E. Furthermore, for any two continuous functions $f$ and $g$ we have

$$
\left[\int f(\lambda) E(d \lambda)\right]\left[\int g(\lambda) E(d \lambda)\right]=\int f(\lambda) g(\lambda) E(d \lambda) .
$$

Proof. Let $\delta>0$ be such that $\left|f(\lambda)-f\left(\lambda^{\prime}\right)\right|<\epsilon$ if $\left|\lambda-\lambda^{\prime}\right|<2 \delta$, and and let $\pi=\left(\sigma_{i}, \lambda_{i}\right), \pi^{\prime}=\left(\sigma_{j}^{\prime}, \lambda_{j}^{\prime}\right)$ be two partitionings of $\sigma$ with norms at most $\delta$. Then for $x \in \mathfrak{X}$ and $x^{*} \in \mathfrak{X}^{*}$, and the operator

$$
U(\pi) \equiv \Sigma f\left(\lambda_{i}\right) E\left(\sigma_{i}\right)
$$

we have the inequality

$$
\left|x^{*}\left(U(\pi)-U\left(\pi^{\prime}\right)\right) x\right| \leq \sum_{i} \sum_{j}\left|f\left(\lambda_{i}\right)-f\left(\lambda_{j}^{\prime}\right)\right|\left|x^{*} E\left(\sigma_{i} \sigma_{j}^{\prime}\right) x\right| \leq \underset{\sigma}{\operatorname{var}} x^{*} E(\sigma) x .
$$


But

$$
\underset{\sigma}{\operatorname{var}} x^{*} E^{\prime}(\sigma) x \leq \underset{\sigma}{4} \operatorname{l.u}_{\sigma}\left|x^{*} E(\sigma) x\right| \leq 4 K|x|\left|x^{*}\right|
$$

where $K$ is an upper bound for $|E(\sigma)|$. Thus

$$
\left|U(\pi)-U\left(\pi^{\prime}\right)\right|=\underset{|x|=\left|x^{*}\right|=1}{\text { l.u.b. }}\left|x^{*}\left(U(\pi)-U\left(\pi^{\prime}\right)\right) x\right| \leq 4 \in K^{\prime} \text {. }
$$

The final assertion is seen by using $(\alpha)$ to obtain the equation

$$
\left[\sum f\left(\lambda_{i}\right) E\left(\sigma_{i}\right)\right]\left[\sum_{j} f\left(\lambda_{j}\right) E\left(\sigma_{j}\right)\right]=\sum f\left(\lambda_{i}\right) g\left(\lambda_{i}\right) E\left(\sigma_{i}\right)
$$

LEMMA 2. Let $\mathscr{X}$ be a commutative subalgebra of $B(X)$ which contains $I$ and the inverse of any of its elements provided that the inverse exists as an element of $B(X)$. Let $T, E \in \mathfrak{U}, E^{2}=E$, and let $N=(m)$ be the set of maximal ideals in 2 . Then ${ }^{3}$

$$
\sigma(T, E X)=[\lambda \mid \lambda=T(m), m \in \mathbb{M}, E(m)=1]
$$

Proof. The symbol $B(X)$, as always, is used for the algebra of all bounded linear operators in the space $\mathcal{X}$. It is normed by the bound of the operator. For an element $T_{0}$ of an algebra 界 0 with unit $E_{0}$, we write $\sigma\left(T_{0}, \Re_{0}\right)$ for the spectrum of $T_{0}$ as an element of $\mathfrak{Y}_{0}$. This is the complement of the set of those $\lambda$ for which $\lambda E_{0}-T_{0}$ has an inverse in $\mathscr{U}_{0}$. According to our hypothesis, then, we have

$$
\sigma(T, \mathfrak{Y})=\sigma(T, \mathfrak{X})=\sigma(T)
$$

I.et $\mathscr{U}_{E}=\mathscr{U} E$, and note that this is a subalgebra of $\mathscr{U}$ with unit $E$. Each $V \in \mathscr{U} E$ maps $E X$ into itself and as an operator in $E X$ has the spectrum $\sigma(V, E X)$. Just as in (i) above we have

$$
\sigma\left(V, \mathfrak{Z} \mathfrak{Z}_{E}\right)=\sigma(V, E \mathfrak{X}) \text {. }
$$

To see this, let

$$
V_{0}=(\lambda I-V) E \in \mathfrak{U}_{E},
$$

${ }^{3}$ The difference algebra $\mathfrak{U}-m$ is the complex number system [8]. We write, using Gelfand's notation, $U(\mathrm{~m})$ for the complex number corresponding to an element $U \in \mathcal{U}$ under the natural homomorphism of $\mathcal{U}$ onto $\mathcal{Z} Y-m$. 
and suppose that $V_{0}$ has an inverse as an operator in $E X$. Define

$$
W=V_{0} E+E^{\prime},
$$

so that $\mathbb{W}^{-1} \in B(\mathfrak{X})$. Thus $\mathbb{W}^{-1} \in \mathfrak{A}, \mathbb{W}^{-1} E \in \mathfrak{N} E$; and since $V_{0} \mathbb{W}^{-1} E=E$ it is seen that $V_{0}$ has an inverse as an element of $\mathfrak{A}_{E}$. This proves that $\rho(V, E X) \subset$ $\rho\left(V, \mathfrak{U}_{E}\right)$. The converse inequality being obvious, we have proved (ii). Now let $\mathbb{M}=(m)$ be the set of maximal ideals in $\mathfrak{X}$, and $\mathbb{M}_{E}=\left(m_{E}\right)$ the set of maximal ideals in $\mathfrak{A}_{E}$. We shall next show that

$$
\mathbb{M}_{E}=[m E \mid m \in \mathbb{M}, E(m)=1] ;
$$

that is, the maximal ideals in $\mathfrak{A}_{E}$ are precisely those of the form $m_{E}=m E$, where $m$ is a maximal ideal in $m$ for which $E(m)=1$. Since $E^{2}=E$, we have $E(m)$ always 0 or 1 , and so the statement $E(m)=1$ is equivalent to the statement $E \notin m$. To prove (iii), let $m$ be a maximal ideal in 2 with $E \notin m$. The set $m_{E}=m E$ is clearly a proper ideal in $\mathfrak{R}_{E}$. To see that $m_{E}$ is maximal, let $n_{E}$ be a proper ideal in $\mathfrak{U}_{E}$ which contains $m_{E}$, and let $n$ be the set of all $V \in \mathfrak{U}$ for which $V E \in n_{E}$. Then $n$ is a proper ideal in $\mathscr{C}$ which contains $m$. Since $m$ is maximal, we have $m=n$ and hence $m_{E}=n_{E}$. Conversely, let $m_{E}$ be a maximal ideal in $\mathfrak{A}_{E}$; then $m=m_{E}+\mathfrak{C E} E^{\prime}$ is a proper ideal in $\mathscr{A}$ with $m E=m_{E}$. To see that $m$ is maximal, suppose that $n$ is a proper ideal in $\mathscr{U}$ containing $m$ properly. Then we shall show that $n_{E}=n E$ is a proper ideal in $\mathfrak{U}_{E}$ which contains $m_{E}$ properly. Let $U \in n, U \notin m$. Then $U E \in n_{E}$. Since $E^{\prime} \in m$, we have $U E^{\prime} \in m$ and hence $U E \notin m$. Therefore, since $m_{E} \subset m$, we have $U E \notin m_{E}$, and this proves (iii). Thus we may say that for any $m \in \mathbb{M}$ for which $E(m)=1$ the difference algebras $\mathfrak{X}-m$, $\mathscr{U} E-m E$ are both isometrically isomorphic to the complex number system. There are, therefore, uniquely determined complex numbers $T(m), T E(m E)$ for which

$$
T-T(m) I \in m, T E-(T E)(m E) E \in m E .
$$

From the first of these relations it follows that $T E-T(m) E \in m E$, and from the second, therefore, that $T(m)=(T E)(m E)$. But as $m$ varies over all points in $M$ for which $E(m)=1$, we see from (iii) that $m E$ varies over all maximal ideals in $A E$ and hence $(T E)(m E)=T(m)$ varies over the spectrum of $T E$ as an element of $2 E$. Hence the desired conclusion follows from (ii).

DEFINITION 1. An operator $S$ is said to be of scalar type in case it is a spectral operator and satisfies the equation 


$$
S=\int \lambda E(d \lambda)
$$

where $E$ is the resolution of the identity for $S$. According to Theorem $1, S(e)=0$ if $e \subset \rho(S)$ so that the integral over the compact set $\sigma(S)$ exists in the uniform topology of operators.

THEOREM 8. An operator $T$ is a spectral operator of class ( $\Gamma$ ) if and only if it is the sum $T=S+N$ of a scalar type operator $S$ of class ( $\Gamma$ ) and a generalized nilpotent operator $N$ commuting with $S$. Furthermore, this decomposition is unique and $T$ and $S$ have the same spectrum and the same resolution of the identity.

Proof. We shall first show that the sum $T=S+N$ of an arbitrary spectral operator $S$ of class $(\Gamma)$ and a generalized nilpotent $N$ commuting with $S$ is itself a spectral operator of class $(\Gamma)$. Let $E$ be the resolution of the identity for $S$, and let $\sigma$ be a Borel set of complex numbers. Then, by Theorem $5, N E(\sigma)=$ $E(\sigma) N$. Let $\mathscr{X}$ be the smallest commutative subalgebra of $B(X)$ containing $N$, $S, E(\sigma), I$, and also containing the inverse of any of its elements provided that the inverse exists as an element in $B(X)$. Then, as established in equation (ii) during the proof of Lemma 2, we have

$$
\sigma(S+N, E(\sigma) X)=\sigma(S+N, \text { } \mathscr{X} E(\sigma)) .
$$

Thus if $M(\sigma)$ is the set of maximal ideals in $A E(\sigma)$, we have

$$
\begin{aligned}
\sigma(T, E(\sigma) \mathfrak{X}) & =[\lambda \mid \lambda=S(m)+N(m), m \in \mathbb{M}(\sigma)] \\
& =\left[\lambda \mid \lambda=S(m), m \in \mathbb{M}_{\sigma}\right] \\
& =\sigma(S, \mathscr{U} E(\sigma))=\sigma(S, E(\sigma) X) \subset \bar{\sigma} .
\end{aligned}
$$

Thus $T$ is a spectral operator of class $(\Gamma)$, and its resolution of the identity is also $E$. Conversely, let $T$ be a spectral operator of class $(\Gamma)$ with resolution of the identity $E$. Using Theorem 7, define

$$
S=\int \lambda E(d \lambda), N=T-S
$$

Clearly $S$ and $N$ commute. It will first be shown that $N$ is a generalized nilpotent. Let $\mathfrak{A}$ be the algebra generated by $T, E(\sigma)(\sigma$ a Borel set $), N$, $I$, and with the property that $U^{-1} \in \mathfrak{U}$ if $U \in \mathfrak{U}$ and $U^{-1} \in B(\mathfrak{X})$. Let $\mathbb{P}=(m)$ be the set of maximal ideals in $\mathfrak{A}$. Then $E(\delta)(\mathrm{m})$ is a zero-one valued additive set function, and hence determines uniquely a complex number $\lambda(m)$ with the property 
that $E\left(\delta_{m}\right)(m)=1$ provided that $\delta_{m}$ is a neighborhood of $\lambda(m)$. Thus for every neighborhood $\delta_{m}$ of $\lambda(m)$ we have

$$
S(m)=\int \lambda E(d \lambda)(m)=\int_{\delta_{m}} \lambda E(d \lambda)(m)=\lambda(m) .
$$

Since $E\left(\delta_{m}\right)(m)=1$ if $\delta_{m}$ is a neighborhood of $\lambda(m)$, it follows from Lemma 2 that

$$
T(m) \in \sigma\left(T, E\left(\delta_{m}\right) \chi\right) \subset \delta_{m},
$$

and hence

$$
T(m)=\lambda(m)=S(m), \quad N(m)=0
$$

Thus by a theorem of Gelfand ${ }^{4}, N$ is a generalized nilpotent. It will next be shown that $S$ is a scalar type operator. For this it is sufficient to show that $E$ is the resolution of the identity for $S$. According to Lemma 2,

$$
\begin{aligned}
\sigma(S, E(\delta) \mathfrak{X}) & =[\lambda \mid \lambda=S(m), m \in \mathbb{M}, E(\delta)(m)=1] \\
& =[\lambda \mid \lambda=T(m), m \in \mathbb{M}, E(\delta)(m)=1] \\
& =\sigma(T, E(\delta) \mathfrak{X}) \subset \bar{\delta},
\end{aligned}
$$

and this shows that $E$ is the resolution of the identity for $S$. Finally it remains to be shown that $S$ and $N$ are uniquely determined by $T$. Let $T=S_{1}+N_{1}$, where $S_{1}$ is of scalar type and $N_{1}$ is a generalized nilpotent commuting with $S_{1}$. Let $E_{1}$ be the resolution of the identity for $S_{1}$. Then, by Theorem 5 ,

$$
N_{1} E_{1}(\sigma)=E_{1}(\sigma) N_{1}
$$

so that $E_{1}(\sigma)$ commutes with $T$. It was established in (*) above that $\sigma(T$, $\left.E_{1}(\sigma) \mathcal{X}\right) \subset \bar{\sigma}_{\text {g }}$ and hence $E_{1}$ is a resolution of the identity. By Theorem 6 , we have $E(\sigma)=E_{1}(\sigma)$, and hence $S=S_{1}, N=N_{1}$.

DEFinition 2. The decomposition, given in Theorem 8, of a spectral operator $T=S+N$ into a sum of a scalar type operator $S$ and a generalized nilpotent $N$ commuting with $S$ is called the canonical decomposition of $T$. The

\footnotetext{
${ }^{4}$ I. Gelfand [8] has shown that $N$ is a generalized nilpotent if and only if $N$ belongs to every maximal ideal.
} 
operator $S$ is called the scalar part of $T$, and $N$ is called the generalized nilpotent part, or the radical part, of $T$.

IEMMA 3. Let $E$ be the resolution of the identity for the spectral operator $T$, and let $N$ be its radical part. Then in the uniform topology of operators, and uniformly with respect to $\xi$ in any closed set $\rho \subset \rho(T)$, we have

$$
T(\xi)=\sum_{n=0}^{\infty} N^{n} \int \frac{E(d \lambda)}{(\xi-\lambda)^{n+1}}
$$

Proof. By Theorem 7 the integral exists in the uniform operator topology, and

$$
\left|\int \frac{E(d \lambda)}{(\xi-\lambda)^{n+1}}\right| \leq r^{n+1} v(E)
$$

where $r=\max |\xi-\lambda|^{-1}$, the maximum being taken over $\lambda \in \sigma(T), \xi \in \rho$. Since $N$ is a generalized nilpotent,

$$
\sqrt[n]{\left|N^{n}\right|} \rightarrow 0
$$

and hence the series

$$
\sum\left|N^{n}\right| r^{n+1}
$$

converges. Thus the series

$$
U=\sum_{n=0}^{\infty} N^{n} \int \frac{E(d \lambda)}{(\xi-\lambda)^{n+1}}
$$

converges in the uniform operator topology, and uniformly with respect to $\xi \in \rho$. From Theorem 7 we have

$$
\left.(\xi I-S) \int \frac{E(d \lambda)}{(\xi-\lambda)^{n+1}}=\left[\int(\xi-\lambda) E(d \lambda)\right] \iint \frac{E(d \lambda)}{(\xi-\lambda)^{n+1}}\right]=\int \frac{E(d \lambda)}{(\xi-\lambda)^{n}}
$$

and so, if $S$ is the scalar part of $T$,

$$
(\xi I-T) U=(\xi I-S-N) \sum_{0}^{\infty} N^{n} \int \frac{E(d \lambda)}{(\xi-\lambda)^{n+1}}
$$




$$
=\sum_{0}^{\infty}\left\{N^{n} \int \frac{E(d \lambda)}{(\xi-\lambda)^{n}}-N^{n+1} \int \frac{E(d \lambda)}{(\xi-\lambda)^{n+1}}\right\}=I .
$$

This proves the lemma.

THEOREM 9. Let $T$ be a spectral operator and $N$ its radical part. Then for every scalar function $f$ analytic and single-valued on the spectrum $\sigma(T)$ we have, in the uniform topology of operators,

$$
f(T)=\sum_{n=0}^{\infty} \frac{N^{n}}{n !} \int f^{(n)}(\lambda) E(d \lambda) .
$$

Proof. Let $C$ be an admissible rectifiable Jordan curve in $\rho(T)$ containing $\sigma(T)$ in its interior and such that $f$ is analytic on and within $C$. Then, using Lemma 3, we have

$$
\begin{aligned}
f(T) & =\frac{1}{2 \pi i} \int_{C} f(\xi) T(\xi) d \xi=\sum_{n=0}^{\infty} N^{n} \int_{C} f(\xi) \int_{\sigma(T)} \frac{E(d \lambda)}{(\xi-\lambda)^{n+1}} \\
& =\sum_{n=0}^{\infty} N^{n} \int_{\sigma(T)}\left[\int_{C} \frac{f(\xi) d \xi}{(\xi-\lambda)^{n+1}}\right] E(d \lambda) \\
& =\sum_{n=0}^{\infty} \frac{N^{n}}{n !} \int_{\sigma(T)} f^{(n)}(\lambda) E(d \lambda) .
\end{aligned}
$$

DEFinition 3. An operator $T$ is said to be of type $m$ in case it is a spectral operator with resolution of the identity $E$ and

$$
f(T)=\sum_{n=0}^{m} \frac{N^{n}}{n !} \int f^{(n)}(\lambda) E(d \lambda), \quad f \in F(T) .
$$

THEOREM 10. Let $N$ be the radical part of the spectral operator $T$; then $T$ is of type $m$ if and only if $N^{m+1}=0$.

Proof. If $N^{m+1}=0$ then clearly the formula of Theorem 9 reduces to that of Definition 3. Conversely, if $T$ is of type $m$ we see, by placing

$$
f(\lambda)=\lambda^{m+1} /(m+1) !
$$


in these two formulas, that

$$
0=N^{m+1} \int E(d \lambda)=N^{m+1}
$$

COROLLARY. A spectral operator is of scalar type if and only if it is of type 0.

We shall next endeavor to characterize operators of finite type in terms of the rate of growth of the resolvent. To this end we introduce the following definition.

DEFINITION 4. Let $E$ be the resolution of the identity for the spectral operator T. If $\xi \notin \sigma(T, E(\sigma) X)$, and in particular if $\xi \notin \bar{\sigma}$, the operator $T_{\sigma}(\xi)$ is defined on $\mathcal{X}$ as follows. For each $x$ in $\mathfrak{X}, T_{\sigma}(\xi) x$ is that uniquely determined point $y \in E(\sigma) X$ for which $(\xi I-T) y=E(\sigma) x$. Thus $T_{\sigma}(\xi)$ is a bounded linear operator in $\chi$ formed by first projecting with $E(\sigma)$ and then operating with the inverse of $(\xi I-T)$ in $E(\sigma) x$.

THEOREM 11. In Hilbert space a spectral operator $T$ is of type $m-1$ if and only if there is a constant $K$ such that, for every Borel set $\sigma$,

$$
\left|\operatorname{dis}(\xi, \sigma)^{m} T_{\sigma}(\xi)\right| \leq K, \xi \notin \sigma,|\xi| \leq|T|+1 .
$$

Proof. In view of Theorem 10 it is sufficient to prove that the condition $\left(^{*}\right)$ is equivalent to the condition $N^{m}=0$. If $N^{m}=0$, and $\xi \notin \bar{\sigma}$, then

$$
T_{\sigma}(\xi)=\sum_{n=0}^{m-1} N^{n} \int_{\sigma} \frac{E(d \lambda)}{(\lambda-\xi)^{n+1}},
$$

from which the condition $(*)$ follows.

The converse will require the following lemma.

Lemma 4. Let $T$ be a spectral operator in Hilbert space. $\mathfrak{X}$ and let $E$ be its resolution of the identity. Then there is a constant $M$ such that for any finite collection $A_{j}(j=1,2, \cdots, n)$ of bounded operators in $\mathfrak{X}$ which commute with $T$, and any collection $\sigma_{i}(j=1,2, \cdots, n)$ of disjoint Borel sets, we have

$$
\left|\sum_{j=1}^{n} A_{j} E\left(\sigma_{j}\right)\right| \leq M \sup _{1 \leq j \leq n}\left|A_{j}\right| .
$$


Proof. It is known (see [16]) that there is a linear one-to-one map $B$ with $B \mathfrak{X}=\mathfrak{X}$, with $B$ and $B^{-1}$ both continuous and such that for each Borel set $\sigma$ the projection

$$
P(\sigma)=B E(\sigma) B^{-1}
$$

is self-adjoint. If $B_{j}=B A_{j} B^{-1}$ then

$$
B\left\{\sum_{j=1}^{n} A_{j} E\left(\sigma_{j}\right)\right\} B^{-1}=\sum_{j=1}^{n} B_{j} P\left(\sigma_{j}\right) .
$$

By Theorem 5, $A_{j}$ commutes with $E(\sigma)$ and hence $B_{j}$ commutes with $P(\sigma)$. Thus

$$
\begin{aligned}
\left|\sum_{j=1}^{n} B_{j} P\left(\sigma_{j}\right) x\right|^{2} & =\left|\sum_{j=1}^{n} P\left(\sigma_{j}\right) B_{j} x\right|^{2}=\sum_{j=1}^{n}\left|P\left(\sigma_{j}\right) B_{j} x\right|^{2} \\
& \leq \sup _{j}\left|B_{j}\right|^{2} \sum_{j=1}^{n}\left|P\left(\sigma_{j}\right) x\right|^{2} \leq \sup _{j}\left|B_{j}\right|^{2}|x|^{2},
\end{aligned}
$$

which proves the lemma.

Now let $T=S+N$ be the canonical form of the spectral operator $T$ which we assume enjoys the property $(*)$ of the theorem. Since

$$
(T-\xi I)^{m}=(S+N-\xi I)^{m}=\sum_{r=0}^{m}\left(\begin{array}{l}
m \\
r
\end{array}\right)(S-\xi I)^{m-r} N^{r},
$$

and

$$
\int_{\sigma(T)}(S-\xi I)^{p} E(d \xi)=0
$$

we have

$$
N^{m}=\int_{\sigma(T)}(T-\xi I)^{m} E(d \xi) .
$$

Now let $\sigma(T)$ be partitioned into the Borel sets $\sigma_{j} \quad(j=1,2, \cdots, n(\delta))$, each of diameter at most $\delta>0$, and let $\xi_{j} \in \sigma_{j}(j=1,2, \cdots, n(\delta))$. Let $C_{j}$ be the circle with center $\xi_{j}$ and radius $2 \delta$. Then since the distance from a point $\lambda$ on $C_{j}$ to 
$\sigma_{j}$ is at least $\delta$ we have

$$
\left|\left(\lambda-\xi_{j}\right)^{m} T_{\sigma_{j}}(\lambda)\right| \leq 2^{m} K, \lambda \in C_{j} .
$$

Let

$$
\lambda_{k}^{(j)}=\xi_{j}+2 \delta \exp (2 k \pi i / p),
$$

so that

$$
\begin{aligned}
\sum_{j=1}^{n(\delta)}\left(T-\xi_{j} I\right)^{m} E\left(\sigma_{j}\right) & =\sum_{j=1}^{n(\delta)} \frac{1}{2 \pi i} \int_{C_{j}}\left(\lambda-\xi_{j}\right)^{m} T_{\sigma_{j}}(\lambda) d \lambda \\
& =\lim _{p} \sum_{j=1}^{n(\delta)} \frac{1}{2 \pi i}\left\{\sum_{k=1}^{p}\left(\lambda_{k}^{(j)}-\xi_{j}\right)^{m} T_{\sigma_{j}}\left(\lambda_{k}^{(j)}\right)\left(\lambda_{k}^{(j)}-\lambda_{k-1}^{(j)}\right)\right\} E\left(\sigma_{j}\right) .
\end{aligned}
$$

But

$$
\begin{aligned}
\sup _{j} \mid \frac{1}{2 \pi i} \sum_{k=1}^{p} & \left(\lambda_{k}^{j}-\xi_{j}\right)^{m} T_{\sigma_{j}}\left(\lambda_{k}^{j}\right)\left(\lambda_{k}^{(j)}-\lambda_{k-1}^{(j)}\right) \mid \\
& \leq 2^{m-1} K \pi^{-1} \sup _{j} \sum_{k=1}^{p}\left|\lambda_{k}^{(j)}-\lambda_{k-1}^{(j)}\right|<2^{m+1} K \delta,
\end{aligned}
$$

and by Lemma 4 therefore

$$
\left|\sum_{j=1}^{n(\delta)}\left(T-\xi_{j} I\right)^{m} E\left(\sigma_{j}\right)\right| \leq 2^{m+1} M K \delta
$$

which shows that

$$
N^{m}=\int(T-\xi I)^{m} E(d \xi)=0 .
$$

THEOREM 12. In Hilbert space a spectral operator $T$ whose spectrum is nowhere dense is of type $m-1$ if and only if its resolvent has at most $m$ th order rate of growth for $\xi$ near the spectrum.

Proof. This theorem is an immediate corollary of Theorem 11. 
4. Algebras of spectral operators. In this section we shall characterize commutative algebras of spectral operators. To this end we shall need the following preliminary lemmas.

LEMMA 5. If $T$ is of class ( $\Gamma)$ with resolution of the identity $E(T)$, and $f \in F(T)$, then $f(T)$ is of class $(\Gamma)$ and its resolution of the identity is given by the formula

$$
E(f(T), \sigma)=E\left(T, f^{-1}(\sigma)\right)
$$

Proof. The foregoing formula clearly yields a spectral measure commuting with $f(T)$. Also $x^{*} E(f(T), \sigma) x$ is countably additive if $x^{*} \in \Gamma$. Now if $\lambda_{0} \notin \bar{\sigma}$ then the function

$$
h(\lambda) \equiv \frac{1}{\lambda_{0}-f(\lambda)}
$$

is analytic on the closure of $f^{-1}(\sigma)$ and hence if $C$ is an admissible contour surrounding the closure of $f^{-1}(\sigma)$ we have

$$
\left(\frac{1}{2 \pi i} \int_{C} h(\lambda) T_{f^{-1}(\sigma)}(\lambda) d \lambda\right)\left(\lambda_{0} I-f(T)\right) E\left(T, f^{-1}(\sigma)\right)=E\left(T, f^{-1}(\sigma)\right)
$$

which shows that

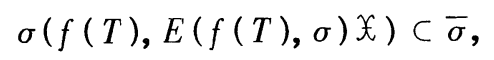

and this completes the proof of the lemma.

At this point we introduce the notion of an integral which will be needed later. For the purposes of the following theorem the Riemann integral will suffice, but for subsequent work the next lemma will be needed for a more general integral. Accordingly let $\mathbb{M}$ be a set, $B$ a field of its subsets with $\mathbb{M} \in B$, and let $B(M)$ be the normed linear space of all complex bounded functions on $\mathbb{M}$ which are measurable $B$. The norm in $B(M)$ is given by $|f|=$ $\sup _{m}|f(m)|$. Let $E$ be an additive operator-valued function on $B$ with

$$
|E(e)| \leq M, \quad e \in \mathbb{B}
$$

For a finitely valued function

$$
f=\sum_{i=1}^{n} \alpha_{i} \psi_{e_{i}} \in \mathbb{B}(\mathbb{M})
$$


we define the integral

$$
\int_{M} f(m) E(d m)=\sum_{i=1}^{n} \alpha_{i} E\left(e_{i}\right)
$$

and note that this definition is independent of the representation of $f$. Also

$$
\begin{aligned}
& \left|\int_{M} f(m) E(d m)\right|=\sup _{|x|=\left|x^{*}\right|=1}\left|\sum \alpha_{i} x^{*} E\left(\sigma_{i}\right) x\right| \\
& \leq \sup _{i}\left|\alpha_{i}\right| \operatorname{var}_{e \in B} x^{*} E(e) x \leq \sup _{i}\left|\alpha_{i}\right| 4 \sup _{e \in B}\left|x^{*} E(e) x\right| \leq 4 M \sup _{m}|f(m)| .
\end{aligned}
$$

Thus if $f \in \mathbb{B}(\mathbb{M})$ is the limit in $B(\mathbb{M})$ of two sequences $\left\{f_{n}\right\}$ and $\left\{g_{n}\right\}$ of finitely valued functions in $B(\mathbb{R})$ then

$$
\lim _{n} \int_{\mathbb{M}} f_{n}(m) E(d m)=\lim _{n} \int_{\mathscr{M}} g_{n}(m) E(d m),
$$

and this limit is taken as the definition of the integral

$$
\int_{M} f(m) E(d m)
$$

It is clear that in case $\mathbb{M}$ is a compact set in the plane and $f$ is continuous the integral as defined coincides with the Riemann integral. In case $E$ is a spectral measure on $B$ for which $x^{*} E(e) x$ is countably additive on $B$ for each $x \in X$ and each $x^{*}$ in a total linear manifold $\Gamma \subset X^{*}$, we say that $E$ is a spectral measure of class $(B, \Gamma)$.

LEMMA 6. Let $\mathbb{B}$ be a $\sigma$-field of subsets of a set $\mathbb{M}$ with $\mathbb{M} \in \mathbb{B}$. Let E be a spectral measure of class $(B, \Gamma)$, and for $f \in B\left(\mathbb{M}^{\prime}\right)$ let

$$
S(f) \equiv \int_{M} f(m) E(d m) \text {. }
$$

Then there is a constant $v(E)$ such that

$$
|S(f)| \leq v(E)|f|, \quad f \in \mathbb{B}(\mathbb{M})
$$

Also for every $f \in B(M)$ the operator $S(f)$ is a scalar type operator of class $(\Gamma)$ whose resolution of the identity $E(S)$ is given by the equation 


$$
E(S, e)=E\left(f^{-1}(e)\right)
$$

Proof. The first conclusion follows from the foregoing definition of the integral. Now if $E$ is a spectral measure the map $f \rightarrow S(f)$ of $B(\stackrel{m}{)})$ into $B(X)$ is a homomorphism; that is, it preserves multiplication as well as addition. Thus, if $\lambda_{0} \notin \bar{\sigma}$, the operator

$$
U=\int_{\mathscr{M}}\left(\lambda_{0}-f(m)\right)^{-1} \psi_{f^{-1}(\sigma)}(m) E(d m)
$$

satisfies the equation

$$
\left(\lambda_{0} I-S(f)\right) U=E\left(f^{-1}(\sigma)\right)
$$

which shows that

$$
\sigma\left(S(f), E\left(f^{-1}(\sigma)\right) \mathfrak{X}\right) \subset \bar{\sigma} .
$$

Thus $S$ is a spectral operator whose resolution of the identity is given by

$$
E(S, \sigma)=E\left(f^{-1}(\sigma)\right)
$$

To see that $S$ is a scalar type operator we decompose the closure of $f(\mathbb{M})$ into a finite number of disjoint parts $\sigma_{i}$, each of diameter at most $\epsilon$. Let $\lambda_{i} \in \sigma_{i}$. Then

$$
\left|\sum \lambda_{i} \psi_{\sigma_{i}}(f(m))-f(m)\right|<\epsilon, \quad m \in \mathbb{M},
$$

and so

$$
S(f)=\lim _{\epsilon \rightarrow 0} \sum \lambda_{i} E\left(f^{-1}\left(\sigma_{i}\right)\right)=\lim _{\epsilon \rightarrow 0} \int \sum_{i} \lambda_{i} \psi_{\sigma_{i}}(\lambda) E(S, d \lambda)=\int \lambda E(S, d \lambda),
$$

which proves that $S$ is of scalar type.

Definition 5. If $T, U, \cdots, V$ are in $B(X)$, the symbol $\mathcal{U}(T, U, \cdots, V)$ will stand for the smallest subalgebra of $B(X)$ which is closed in the norm topology of $B(X)$, which contains $T, U, \ldots, V$, and $I$, and which contains the inverse $W^{-1}$ of any of its elements provided that the inverse exists as an element of $B(\mathfrak{X})$. The algebra $\mathcal{U}(U, T, \cdots, V)$ will sometimes be called the full algebra generated by $U, T, \ldots, V$. If $\sigma$ is a compact set in the complex plane, 
the symbol $C R(\sigma)$ will stand for the algebra of all complex functions $f(\lambda)$, $\lambda \in \sigma$ which may be approximated uniformly on $\sigma$ by rational functions. The norm in $C R(\sigma)$ is

$$
|f|=\max _{\lambda \in \sigma}|f(\lambda)|,
$$

so that $C R(\sigma)$ is a subalgebra of $C(\sigma)$. Two $B$-algebras are said to be equivalent in case they are topologically and algebraically isomorphic.

THEOREM 13. Let $T$ be a spectral operator and $S$ its scalar part. Then, as a vector direct sum,

$$
\mathfrak{U}(T, S)=\mathfrak{U}(S) \oplus \Re,
$$

where $\mathfrak{Y}$ is the radical in $\mathfrak{X}(T, S)$. Furthermore, $\mathfrak{X}(S)$ is equivalent to $C R(\sigma(T))$, and every operator in $\mathfrak{A}(T, S)$ is a spectral operator.

Proof. If $f$ is rational and analytic on $\sigma(T)=\sigma(S)$, then $f(\sigma(S))=\sigma(f(S))$ and thus

$$
\max _{\lambda \in \sigma(S)}|f(\lambda)| \leq|f(S)| \leq \max _{\lambda \in \sigma(S)}|f(\lambda)| v(E) .
$$

Thus $\mathfrak{U}(S)$ is equivalent to $C R(\sigma(S))$. Since $\mathscr{U}(S)$ has no radical it is seen that $\mathfrak{X}(S) \oplus \mathfrak{H}$ is a direct vector sum contained in $\mathfrak{A}(T, S)$. Now let $N$ be the radical part of $T$. It follows from Theorems 8 and 9 and Lemma 6 that the canonical decomposition of $f(T)$ for $f \in F(T)$ is

$$
f(T)=f(S)+N_{1} \text {. }
$$

Hence in particular if $T^{-1}$ exists its canonical decomposition is

$$
T^{-1}=S^{-1}+N_{2}
$$

Also

$$
T^{n}=S^{n}+N_{3}, \quad T^{n} S^{m}=S^{n+m}+N_{4},
$$

and thus for a polynomial $P$ in $T$ and $S$ we have

$$
P(T, S)=Q(S)+N_{5},
$$


where $Q$ is a polynomial and $N_{5}$ a generalized nilpotent. Since for $m$ in the space M of maximal ideals of $\mathfrak{X}(T, S)$ we have

$$
P(S(m), S(m))=P(T(m), S(m))=Q(S(m)),
$$

it is seen that $Q(S)=P(S, S)$ and thus

$$
P(T, S)=P(S, S)+N_{5} \text {. }
$$

If $P_{1}$ is also a polynomial in two variables, the operator

$$
R(T, S)=P(T, S) P_{1}(T, S)^{-1}
$$

will be defined as an element of $\mathscr{X}(T, S)$ if and only if $P_{1}(\lambda, \lambda) \neq 0$ for $\lambda \in \sigma(T)$. In this case we see from (i) and (ii) that

$$
R(T, S)=R(S, S)+N_{6} \text {. }
$$

Since $R(S, S)$ is of type 0 , this is the canonical form for $R(T, S)$. An arbitrary $U \in \mathscr{U}(T, S)$ is a limit, $U=\lim R_{n}$, of rational functions $R_{n}$ in $T$ and $S$. Since

$$
\sigma(T)=\sigma(S)=S(\mathbb{M})=T(\mathbb{M}),
$$

and $T(m)=S(m)$, we have

$$
\begin{aligned}
\sup _{\lambda \in \sigma(S)}\left|R_{n}(\lambda, \lambda)-R_{p}(\lambda, \lambda)\right|=\sup _{m} & \left|\left\{R_{n}(T, S)-R_{p}(T, S)\right\}(m)\right| \\
& \leq\left|R_{n}(T, S)-R_{p}(T, S)\right| \rightarrow 0 .
\end{aligned}
$$

Hence $R_{n}(\lambda, \lambda)$ converges uniformly on $\sigma(S)$ to a function $f \in C R(\sigma(S))$. Thus $R_{n}(S, S) \rightarrow f(S)$ in $\mathscr{U}(S)$, and $U \in \mathfrak{U}(S) \oplus \Re$. It follows from Lemma 6 that every operator in $\mathfrak{U}(S)$ is a scalar type operator and thus it is seen, by Theorem 8 , that every operator in $\mathcal{Q}(T, S)$ is a spectral operator.

THEOREM 14. Let $E$ be the resolution of the identity of the spectral operator $T$. Let $\mathbb{M}$ be the space of maximal ideals is the algebra

$$
\mathfrak{U}=\mathfrak{U}(E(\sigma), \sigma \text { a Borel set }) .
$$

Let $R_{1}$ be the radical in the algebra

$$
\mathfrak{W}_{1}=\mathfrak{U}(T, E(\sigma), \sigma \text { a Borel set }) .
$$


Then 2 is equivalent to $C(\stackrel{m}{m)}$, and

$$
\mathfrak{H}_{1}=\mathfrak{A} \oplus \Re_{1} \text {. }
$$

Furthermore, every operator in $\mathfrak{X}_{1}$ is a spectral operator.

Proof. Elements of the form

$$
U=\sum_{i=1}^{n} \alpha_{i} E\left(\sigma_{i}\right), E\left(\sigma_{i}\right) \neq 0, \quad \sigma_{i} \sigma_{j}=\varnothing, i \neq j, \underset{i}{\bigcup} \sigma_{i}=\sigma(T)
$$

are dense in 20 since if such an element has an inverse the inverse is again of the same form. Furthermore, if $E\left(\sigma_{i}\right) \notin m \in S M$ then $U(m)=\alpha_{i}$. Thus, using Lemma 6, we have

$$
\sup _{i n}|U(m)|=\sup _{i}\left|\alpha_{i}\right| \leq|U| \leq \sup _{i}\left|\alpha_{i}\right| v(E)=\sup _{m}|U(m)| v(E),
$$

and therefore

$$
\sup _{m}|U(m)| \leq|U| \leq \sup _{m}|U(m)| v(E), \quad U \in \mathfrak{R},
$$

which shows that $\mathscr{U}$ is equivalent to a subalgebra of $C(\mathscr{M})$. Since the projections $E(\sigma)$ generate $\mathfrak{L}$, they distinguish between points in $M$. Also it is clear that the element

$$
V=\sum \bar{\alpha}_{i} E\left(\sigma_{i}\right)
$$

is related to the operator $U$ given in (i) by

$$
\overline{U(m)}=V(m), \quad m \in \mathbb{M} \text {. }
$$

Thus, by the Stone-Weierstrass theorem, $\mathscr{Z}$ is equivalent to $C(\mathfrak{M})$. Hence $\mathfrak{U} \oplus \Re$ is a vector direct sum and a subalgebra of $\mathfrak{U}_{1}$. It is also closed in $\mathfrak{U}_{1}$ since if $m_{1}$ is a maximal ideal in $\mathscr{N}_{1}$ we have, for an arbitrary operator $U=S+N$ with $S \in \mathfrak{R}, N \in \Re$,

$$
|S| v(E)^{-1} \leq \sup _{m_{1}}\left|S\left(m_{1}\right)\right|=\sup _{m}\left|U\left(m_{1}\right)\right| \leq|U| \leq|S|+|N|
$$

Also since $\mathfrak{U} \simeq C(\mathbb{M})$ it is seen that $\mathscr{U} \oplus \Re_{1}$ is a full algebra of operators; 
that is, it contains the inverse $\mathbb{W}^{-1}$ of any of its elements provided that $\mathbb{W}^{-1}$ exists as an element of $B(\mathfrak{X})$. Thus $\mathfrak{X}_{1} \subset \mathfrak{U} \oplus \mathfrak{R}_{1} \subset \mathfrak{R}_{1}$. Finally, to see that every operator in $\mathfrak{U}_{1}$ is a spectral operator it will, in view of Theorem 8 , suffice to show that every $U \in \mathfrak{2}$ is a scalar type operator. Consider a finitely valued measurable function

$$
f(\lambda)=\sum \alpha_{i} \psi_{\sigma_{i}}(\lambda), \quad \lambda \in \sigma(S)
$$

We may suppose that $\sigma_{i} \sigma_{j}=\varnothing \quad(i \neq j)$, and $U_{\sigma_{i}}=\sigma(S)$, so that the values of $f$ are the numbers $\alpha_{i}$. The operator

$$
f(S)=\int_{\sigma(S)} f(\lambda) E(d \lambda)=\sum_{i} \alpha_{i} E\left(\sigma_{i}\right),
$$

as was shown above, has the property that except for $\lambda$ in a set $\sigma$ with $E(\sigma)=0$ we have $|f(\lambda)| \leq f(S)$. Thus if we define the norm

$$
|f|_{E} \equiv E \text {-ess. sup }|f(\lambda)| \equiv \inf _{E(\sigma)=I} \sup _{\lambda \in \sigma}|f(\lambda)| \text {, }
$$

the operator $f(S)$ satisfies the inequality

$$
|f|_{E} \leq|f(S)| \leq|f|_{E} v(E)
$$

The general operator $U$ in $\mathscr{V}$ is the limit of a sequence $f_{n}(S)$, where $f_{n}(\lambda)$ is a finitely. valued measurable function. Thus

$$
f(\lambda)=\lim f_{n}(\lambda)
$$

exists uniformly except on a set $\sigma \subset \sigma(S)$, where $E(\sigma)=0$, and

$$
U=\int_{\sigma(S)} f(\lambda) E(d \lambda) .
$$

Hence, by Lemma $6, U$ is a scalar type operator.

DEFINITION 6. If $T=S+N$ is the canonical decomposition of the spectral operator $T$, and $E$ is its resolution of the identity, by $E B(\sigma(T))$ will be meant the space of all $E$-essentially bounded Borel measurable functions defined on $\sigma(T)=\sigma(S)$. The norm is 


$$
|f|=E \text {-ess. } \sup _{\lambda \in \sigma(S)}|f(\lambda)| \equiv \inf _{E(\sigma)=I} \sup _{\lambda \in \sigma}|f(\lambda)|
$$

According to what has just been shown we may state:

THEOREM 15. In the notation of Theorem 14 we have $\mathcal{A}$ equivalent to $E B(\sigma(T))$.

THEOREM 16. If $S$ is a scalar type operator with resolution of the identity $E$, and $f$ is an E-essentially bounded Borel function on $\sigma(S)$, then

$$
\sigma(f(S))=\bigcap_{E(\sigma)=I} \overline{f(\sigma)} .
$$

Proof. If $\lambda_{0} \notin \overline{f(\sigma)}$, where $E(\sigma)=1$, then

$$
h(\lambda)= \begin{cases}\left(\lambda_{0}-f(\lambda)\right)^{-1}, & \lambda \in \sigma, \\ 0, & , \lambda \notin \sigma,\end{cases}
$$

is a bounded Borel measurable function and

$$
h(S)\left(\lambda_{0} I-f(S)\right)=I,
$$

so that $\lambda_{0} \in \rho(f(S))$. Thus $\overline{f(\sigma)} \supset \sigma(f(S))$ if $E(\sigma)=I$, and

$$
\bigcap_{E(\sigma)=I} \overline{f(\sigma)} \supset \sigma(f(S)) .
$$

Conversely, if $\lambda_{0} \in \rho(f(S))$ we see from Theorem 15 that $\left(\lambda_{0}-f(\lambda)\right)^{-1}$ is $E$-essentially bounded on $\sigma(T)$. Hence there is a Borel set $\sigma$ with $E(\sigma)=I$ and

$$
\left|\lambda_{0}-f(\lambda)\right|^{-1} \leq M, \quad \lambda \in \sigma
$$

Hence $\lambda_{0} \notin \overline{f(\sigma)}$. This shows that

$$
\sigma(f(S)) \supset \overline{f(\sigma)} \supset \underset{E(\sigma)=I}{\cap} \overline{f(\sigma)},
$$

and completes the proof. 
THEOREM 17. Let $\mathfrak{2}(\tau)$ be the full algebra generated by a family $\tau$ of commuting spectral operators together with their resolutions of the identity. If the Boolean algebra determined by the resolutions of the identity of the operators in $\tau$ is bounded, then, as a vector direct sum,

$$
\mathfrak{H}(\tau)=\mathfrak{U}_{1} \oplus \Re,
$$

where $\mathfrak{N}$ is the radical in $\mathfrak{Y}(\tau)$ and $\mathfrak{X}_{1}$ is equivalent to the algebra of continuous functions on the space of maximal ideals in $\mathscr{N}(\tau)$.

Proof. Note first that if $T, U \in \tau$ have resolutions of the identity $E(T, \cdot)$, $E(U, \cdot)$, respectively, then for every pair $\sigma, \mu$ of Borel sets in the plane the projections $E(T, \sigma), E(U, \mu)$ commute. This follows from a double application of Theorem 5. Thus the various projections $E(T, \sigma)$ determined by Borel sets $\sigma$ and operators $T \in \tau$ determine a Boolean algebra $\mathfrak{Q}_{0}$, and by assumption there is a constant $M$ with $|E| \leq M$ for $E \in \mathscr{N}_{0}$. We shall first show that there is a constant $K$ such that

$$
\sum_{i=1}^{n}\left|x^{*} E_{i} x\right| \leq K|x|\left|x^{*}\right|, \quad x \in \mathfrak{X}, \quad x^{*} \in \mathfrak{X}^{*},
$$

provided that $E_{i} \in \mathfrak{H}_{0}$ and $E_{i} E_{j}=0$ for $i \neq j$. To see this, let $\left(x^{*} E x\right)_{r}$ be the real part of $x^{*} E x$. Then, if $E_{i} E_{j}=0 \quad(i \neq j)$, we have

$$
\begin{aligned}
\sum\left|\left(x^{*} E_{i} x\right)_{r}\right| & =\Sigma^{\prime}\left(x^{*} E_{i} x\right)_{r}-\Sigma^{\prime \prime}\left(x^{*} E_{i} x\right)_{r} \\
& =\left(x^{*}\left(\Sigma^{\prime} E_{i}\right) x\right)_{r}-\left(x^{*}\left(\Sigma^{\prime \prime} E_{i}\right) x\right)_{r} \leq 2 M|x|\left|x^{*}\right|,
\end{aligned}
$$

where $\Sigma^{\prime}\left(\Sigma^{\prime \prime}\right)$ represents the sum over those $i$ for which $\left(x^{*} E_{i} x\right)_{r} \geq 0(<0)$. Similarly for the imaginary part of $x^{*} E x$. Thus

$$
\sum\left|x^{*} E_{i} x\right| \leq 4 M|x|\left|x^{*}\right|,
$$

which proves (i).

Now consider elements $U \in \mathfrak{U}(\tau)$ of the form

$$
U=S+N,
$$

where 
(iii)

$$
S=\sum_{i=1}^{n} \alpha_{i} E_{i}
$$

with

$$
0 \neq E_{i} \in \mathfrak{A}_{0}, \quad E_{i} E_{j}=0, \quad i \neq j, E_{1}+\cdots+E_{n}=l,
$$

and where $N \in \Re$, the radical of $\mathscr{H}(\tau)$. If $m \in \mathbb{M}$, the space of maximal ideals in $\mathcal{Z}(\tau)$, then in view of (iii) there is an $i$ with $E_{i}(m)=1, E_{j}(m)=0 \quad(j \neq i)$. Thus $\alpha_{i}=U(m)=S(m)$ and

$$
\sup _{i}\left|\alpha_{i}\right|=\sup _{m}|U(m)| \leq|U| \leq|S|+|N|
$$

From (i) and (iii) it is seen that

$$
|S|=\sup _{|x|=\left|x^{*}\right|=1}\left|\sum \alpha_{i} x^{*} E_{i} x\right| \leq \sup _{i}\left|C_{i}\right| K
$$

and hence, by (iv),

$$
K^{-1}|S| \leq|U| \leq|S|+|N|
$$

The inequality ( $\mathrm{v}$ ) shows that if $U_{n}=S_{n}+N_{n}$ is a convergent sequence of operators, each of the form (ii) with $S_{n}$ of the form (iii) and $N_{n} \in \Re$, then $\left\{S_{n}\right\}$ and $\left\{N_{n}\right\}$ are also convergent sequences. Let $\mathscr{P}_{1}$ be the algebra of all limits $S_{0}=\lim _{n} S_{n}$, where $S_{n}$ has the form (iii). Since for the operator (iii) we have, as shown above,

$$
\sup _{m}|S(m)| \leq|S| \leq \sup _{m}|S(m)| \cdot K,
$$

it is seen that $\mathfrak{C}_{1}$ is equivalent to a subalgebra $C$ of $C(\mathbb{M})$, and the Weierstrass theorem ${ }^{5}$ shows that $C=C(\mathbb{R})$. Clearly therefore, $\mathfrak{L}_{1} \oplus \mathfrak{R}$ is a direct sum, is contained in $\mathscr{U}(\mathcal{T})$, contains every $E(T, \sigma)$ with $T \in \tau$ and $\sigma$ a Borel set in the plane, and contains every $T \in \tau$. This last statement, namely that $\tau \subset \mathfrak{U}_{1} \oplus \Re$, follows since the canonical reduction $T=S+N$ has the property that $N \in \Re$ and $S \in \mathscr{Z}_{1}$. To complete the proof it will suffice to show that $\mathscr{U}_{1} \oplus \Re$ is a full algebra; that is, it will suffice to show that if $T \in \mathfrak{U}_{1} \oplus \Re$, and $T^{-1} \in B(\mathfrak{X})$,

${ }^{5}$ As proved by M. H. Stone [13] for real algebras $C(M)$ and by I. Gelfand and G. Silov [9] for complex algebras $C(\mathbb{M})$. 
then $T^{-1} \in \mathscr{U}_{1} \oplus \Re$. Let $T=S+N$ be the canonical form of $T$; then since $T(m)=S(m) \neq 0, m \in \mathbb{M}$, we see that $S^{-1}$ exists and is in $\mathfrak{U}_{1}$ because $S^{-1}(\cdot) \in$ $C(M)$. Thus

$$
T\left(S^{-1}+M\right)=I,
$$

where $M=-T^{-1} N S^{-1} \in \Re$. Thus $\mathscr{U}_{1} \oplus \Re$ is a full algebra containing $\tau$ and every projection $E(T, \sigma)(T \in \tau)$, and hence $\mathfrak{U}(\tau)=\mathfrak{U}_{1} \oplus \Re$.

THEOREM 18. Let $B$ be the Borel sets in the compact Hausdorff space $\stackrel{m}{M}$,

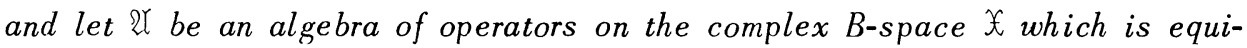
valent to the algebra $C(\mathbb{M})$ of continuous functions on $\mathbb{M}$. Then there is a function $A$ on $B$ to $B\left(\mathfrak{X}^{*}\right)$ with the properties:

(i) A is a spectral measure in $\mathfrak{X}^{*}$ of class $(\mathbb{B}, \mathfrak{X})$;

(ii) if $S(f)$ is the element in $\mathscr{U}$ corresponding to the element $f$ in $C(\mathbb{M})$ under some homeomorphic isomorphism then, for every $x \in \mathfrak{X}$ and $x^{*} \in \mathfrak{X}^{*}$,

$$
x^{*} S(f) x=\int_{\mathfrak{M}} f(m) x A(d m) x^{*}, \quad f \in C(\mathscr{M}) ;
$$

(iii) the adjoint $S^{*}$ of every $S$ in $\mathfrak{X}$ is a scalar type operator of class $\mathfrak{X}$;

(iv) if $\mathfrak{X}$ is reflexive, every $S$ in $\mathcal{X}$ is a scalar type operator of class $\mathfrak{X}^{*}$.

Proof. Let $S(f)$ be the operator in $\mathcal{U}$ corresponding to the function $f \in C(\mathbb{M})$ under some homeomorphic isomorphism of $\mathfrak{X}$ onto $C(\mathscr{M})$. Then for $x$ in $X$ and $x^{*}$ in $\mathfrak{X}^{*}$ we have $x^{*} S(f) x$ a linear functional on $C(\mathbb{N})$ and hence, by the Riesz representation theorem, there is a uniquely determined regular measure $\mu(\cdot$, $\left.x, x^{*}\right)$ such that

$$
x * S(f) x=\int_{M} f(m) \mu\left(d m, x, x^{*}\right), f \in C(\mathbb{M}), x \in \mathfrak{X}, x^{*} \in \mathfrak{X}^{*} .
$$

Since $\mu\left(e, x, x^{*}\right)$ is uniquely determined by $e, x, x^{*}$ it is, for each $e \in B$, bilinear in $x$ and $x^{*}$. Since

$$
\mid \mu\left(e, x, x^{*}\left|\leq \operatorname{var}_{e}\right| \mu\left(e, x, x^{*}\right)\left|=\sup _{|f|=1}\right| x^{*} S(f) x|\leq K| x|| x^{*} \mid\right.
$$

it is seen that $\mu\left(e, x, x^{*}\right)$ is continuous in $x$ and $x^{*}$. Hence for fixed $e$ and $x^{*}$ there is a point $A(e) x^{*} \in \mathfrak{X}^{*}$ such that

$$
\mu\left(e, x, x^{*}\right)=x A(e) x^{*} .
$$


It follows from the bilinearity and boundedness of $\mu$ that $A(e) \in B\left(X^{*}\right)$. Thus (ii) is proved and a part of (i) is proved. To complete the proof of (i) we have, for every pair $f, g \in C(\mathbb{M})$,

$$
\begin{aligned}
\int_{\mathbb{M}} f(m) \int_{\mathbb{M}} g(\mu) x A(d \mu \cap d m) x^{*} & =\int_{\mathbb{M}} f(m) \int_{d m} g(\mu) x A(d \mu) x^{*} \\
& =\int_{\mathbb{M}} f(m) g(m) x A(d m) x^{*}=x^{* S}\left(f_{g}\right) x \\
& =x^{*} S(f) S(g) x=\int_{\mathbb{M}} f(m) S(g) x A(d m) x^{*} \\
& =\int_{\mathbb{M}} f(m) \int_{\mathbb{M}} g(\mu) x A(d \mu) A(d m) x^{*} .
\end{aligned}
$$

Thus, since a functional on $C(\mathscr{N})$ determines the regular measure uniquely, we have

$$
A(\sigma \cap \delta)=A(\sigma) A(\delta), \quad \sigma, \delta \in \mathbb{B},
$$

and this completes the proof of (i).

The integral instead of being thought of as a Lebesgue integral in the weak operator topology may be thought of as an integral in the uniform topology as defined immediately preceding Lemma 6. Thus, by Lemma 6, each of the operators

$$
S^{*}(f)=\int_{\mathfrak{M} l} f(m) A(d m)
$$

is a scalar type operator in $\chi^{*}$ of class $\chi$, which proves (iii). In case $\chi$ is reflexive, $E(\sigma)=A^{*}(\sigma)$ is a spectral measure in $\chi$ and hence, by Lemma 6 ,

$$
S(f)=\int_{M !} f(m) E(d m), \quad f \in C(M),
$$

is a scalar type operator of class $\chi^{*}$, which proves (iv) and completes the proof of the theorem.

THEOREM 19. The adjoint $T^{*}$ of every operator $T$ in the algebra $)(\tau)$ as defined in Theorem 17 is a spectral operator of class $X$. If $X$ is reflexive, every $T \in \mathfrak{Y}(\tau)$ is a spectral operator of class $\mathfrak{X}^{*}$. 
Proof. This follows immediately from Theorems 8, 17, and 18.

Theorem 19 shows that the sum and product of two spectral operators in a reflexive space $\mathfrak{X}$ will again be spectral operators provided that the Boolean algebra determined by all the projections in both resolutions of the identity is bounded. If $\mathfrak{X}$ is Hilbert space, J. W'ermer [16] has shown that such is the case. In general, however, the Boolean algebra determined by two bounded Boolean algebras of projections, all of which commute, is not bounded. Also it is not always true that the sum of two spectral operators is a spectral operator. Examples proving both of these statements have been constructed by S. Kakutani $[10]$.

Examples of spectral operators other than normal operators on Hilbert space are easy to construct, and some interesting classes have been discussed by J. Schwartz [12]. ${ }^{6}$

Besides Theorems 8, 13, 14, 19, which are useful in the construction of spectral operators, we shall mention one more which will be needed in the perturbation theory of J. Schwartz.

THEOREM 20. If $T$ is a compact operator in a reflexive space $\mathfrak{X}$, then $T$ is a spectral operator if and only if the integrals

$$
\frac{1}{2 \pi i} \int_{C} T(\lambda) d \lambda
$$

are bounded as $C$ varies over all admissible contours in the resolvent set. In this case the resolution of the identity is countably additive in the strong operator topology, and the integral (i) is the value of the resolution of the identity on the domain bounded by $C$.

Proof. Let $\lambda_{0}=0, \lambda_{n} \neq 0 \quad(n=1,2, \ldots)$ be the points in the spectrum of T. Let

$$
E\left(\lambda_{n}\right)=\frac{1}{2 \pi i} \int_{C_{n}} T(\lambda) d \lambda \quad(n=1,2, \cdots)
$$

where $C_{n}$ is a circle containing $\lambda_{n}$ but no other spectral point. Since the Boolean

${ }^{6}$ Other spectral operators occurring in analysis will be found in the forthcoming book Spectral Theory by N. Dunford and J. Schwartz. Conditions on the rate of growth of the resolvent which are sufficient to ensure that $T$ be spectral will be found in [4]. 
algebra determined by the $E\left(\lambda_{n}\right)$ is bounded, it may be embedded in a complete ${ }^{7}$ Boolean algebra. We may therefore define

$$
E\left(\lambda_{0}\right)=I-\bigcup_{n=1}^{\infty} E\left(\lambda_{n}\right)
$$

and

$$
E(\sigma)=\underset{\lambda_{n} \in \sigma}{\bigcup} E\left(\lambda_{n}\right)
$$

$\sigma$ arbitrary. If $B$ is the Boolean algebra of all subsets of the plane, it is clear that the map $\sigma \rightarrow F(\sigma)$ is a homomorphism of $B$ onto a Boolean algebra of projections in $\mathfrak{X}$. From our hypothesis it follows that

$$
|E(\sigma)| \leq K, \quad \sigma \in \mathbb{B}
$$

Now let $\sigma_{n} \subset \sigma_{n+1} \subset \cdots$ and $\sigma=\bigcup \sigma_{n}$. Then

$$
E(\sigma)=\underset{\lambda_{n} \in \sigma}{\bigcup} E\left(\lambda_{n}\right)={ }_{n}^{\bigcup} \underset{\lambda_{m} \in \sigma_{n}}{U} E\left(\lambda_{m}\right)={ }_{n}^{\bigcup} E\left(\sigma_{n}\right)
$$

and since $E\left(\sigma_{n}\right) x=x \quad(n \geq m)$ if $x \in E\left(\sigma_{m}\right) \mathcal{X}$, we see from (ii) and (iii)

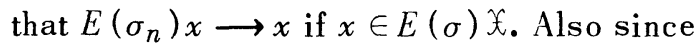

$$
E\left(\sigma^{\prime}\right) \mathfrak{X}=\cap\left(E\left(\sigma_{n}^{\prime}\right) \mathfrak{X}\right)
$$

we have $E\left(\sigma_{n}\right) x=0$ if $x \in E\left(\sigma^{\prime}\right) X$. Thus $E\left(\sigma_{n}\right) x \rightarrow E(\sigma) x$ for every $x$ in $X$, and $E(\sigma)$ is countably additive on $B$ in the strong operator topology. To complete the proof that $T$ is spectral, it will suffice to show that

$$
\sigma(T, E(\sigma) X) \subset \bar{\sigma}, \quad \sigma \in \mathbb{B}
$$

If $\lambda_{0} \notin \bar{\sigma}$, then $E(\sigma)$ has the form (i), from which (iv) follows. If $\lambda_{0} \in \bar{\sigma}$, then any spectral point $\lambda_{n} \notin \bar{\sigma}$ is in $\rho\left(T, E\left(\left\{\lambda_{n}\right\}^{\prime}\right) \mathcal{X}\right)$ and hence in $\rho(T, E(\sigma) X)$, which proves (iv).

Finally let $\sigma$ be an open and closed subset of $\sigma(T)$, and let $A(\sigma)$ be the projection defined by (i), where $\sigma$ is the intersection of $\sigma(T)$ and the domain bounded by $C$. Then, since

\footnotetext{
${ }^{7}$ Complete relative to the order $A \subset B(A B=A)$. See, for example [6].
} 


$$
\sigma(T, A(\sigma) \mathfrak{X}) \subset \sigma, \quad \sigma\left(T, A\left(\sigma^{\prime}\right) \mathfrak{X}\right) \subset \sigma^{\prime},
$$

The orems 3 and 4 show that

$$
E(\sigma) A(\sigma)=A(\sigma), \quad E(\sigma) A\left(\sigma^{\prime}\right)=0,
$$

and hence that

$$
E(\sigma)=E(\sigma)\left(A(\sigma)+A\left(\sigma^{\prime}\right)\right)=A(\sigma)
$$

\section{REFERENCES}

1. W. G. Bade, Unbounded spectral operators, Pacific J. Math. 4 (1954), 373-392. 2. $393-413$. , Weak and strong limits of spectral operators, Pacific J. Math. 4 (1954),

3. N. Dunford, Spectral theory I. Convergence to projections, Trans. Amer. Math. Soc. 54 (1943), $185-217$.

4. (1952), 559-614.

, Spectral theory II. Resolutions of the identity, Pacific J. Math. 2

5. $305-356$.

, Uniformity in linear spaces, Trans. Amer. Math. Soc. 44 (1938),

6. Direct decompositions of Banach spaces, Boletin de la Sociedad Matematica Mexicana, 3 (1946), 1-12.

7. B. Fuglede, A commutativity theorem for normal operators, Proc. Nat. Acad. Sci. 36 (1950), $35-40$.

8. I. Gelfand, Normierte Ringe, Recueil Math. (Mat. Sbornik) 9 (51) (1954), 1-23.

9. I. Gelfand and G. Silov, Über verschiedene Methoden der Einführung der Topologie in die Menge der maximalen Ideale, Recueil Math. (Mat. Sbornik) N. S. 9 (1941), $25-39$.

10. S. Kakutani, An example concerning uniform boundedness of spectral measures, Pacific J. Math. 4 (1954), 363-372.

11. B. J. Pettis, On integration in vector spaces, Trans. Amer. Math. Soc. 44 (1938), 277-304.

12. J. Schwartz, Perturbations of spectral operators, and applications, Pacific J. Math. 4 (1954), 415-458.

13. M. H. Stone, Applications of the theory of Boolean rings to general topology, Trans. Amer. Math. Soc. 41 (1937), $375-481$.

14. A. E. Taylor, Analysis in complex Banach spaces, Bull. Amer. Math. Soc. 49 (1943), $652-669$.

15. (1938), 70-74.

16. J. Wermer, Commuting spectral measures on Hilbert space, Pacific J. Math. 4 (1954), $355-361$. 


\section{PACIFIC JOURNAL OF MATHEMATICS}

\section{EDITORS}

\author{
M.M. SCHIFFE R* \\ Stanford University \\ Stanford, California \\ E. HEWITT \\ University of Washington \\ Seattle 5, Washington
}

R.P. DILWORTH

California Institute of Technology Pasadena 4, California

E.F. BECKENBACH**

University of California

Los Angeles 24, California

\section{ASSOCIATE EDITORS}

$\begin{array}{llll}\text { H. BUSEMANN } & \text { P.R. HALMOS } & \text { BORGE JESSEN } & \text { J. J. STOKER } \\ \text { HERBERT FEDERER } & \text { HEINZ HOPF } & \text { PAUL LÉVY } & \text { E.G. STRAUS } \\ \text { MARSHALL HALL } & \text { R.D. JAMES } & \text { GEORGE PÓLYA } & \text { KÔSAKU YOSIDA }\end{array}$

\section{SPONSORS}

UNIVERSITY OF BRITISH COLUMBIA CALIFORNIA INSTITUTE OF TECHNOLOGY UNIVERSITY OF CALIFORNIA, BERKELEY UNIVERSITY OF CALIFORNIA, DAVIS UNIVERSITY OF CALIFORNIA, LOS ANGELES UNIVERSITY OF CALIFORNIA, SANTA BARBARA UNIVERSITY OF NEVADA OREGON STATE COLLEGE UNIVERSITY OF OREGON
UNIVERSITY OF SOUTHERN CALIFORNIA STANFORD RESEARCH INSTITUTE STANFORD UNIVERSITY WASHINGTON STATE COLLEGE UNIVERSITY OF WASHINGTON

AMERICAN MATHEMATICAL SOCIETY HUGHES AIRCRAFT COMPANY

Mathematical papers intended for publication in the Pacific Journal of Mathematics should be typewritten (double spaced), and the author should keep a complete copy. Manuscripts may be sent to any of the editors. Manuscripts intended for the outgoing editors should be sent to their successors. All other communications to the editors should be addressed to the managing editor, E.G. Straus, at the University of California Los Angeles 24, California.

50 reprints of each article are furnished free of charge; additional copies may be obtained at cost in multiples of 50 .

The Pacific Journal of Mathematics is published quarterly, in March, June, September, and December. The price per volume (4 numbers) is $\$ 12.00$; single issues, $\$ 3.50$; back numbers (Volumes $1,2,3$ ) are available at $\$ 2.50$ per copy. Special price to individual faculty members of supporting institutions and to individual members of the American Mathematical Society: $\$ 4.00$ per volume; single issues, $\$ 1.25$.

Subscriptions, orders for back numbers, and changes of address should be sent to the publishers, University of California Press, Berkeley 4, California.

Printed at Ann Arbor, Michigan. Entered as second class matter at the Post Office, Berkeley, California.

* To be succeeded in 1955, by H.L. Royden, Stanford University, Stanford, California.

** To be succeeded in 1955, by E.G. Straus, University of California, Los Angeles 24, Calif.

UNIVERSITY OF CALIFORNIA PRESS - BERKELEY AND LOS ANGELES 


\section{Pacific Journal of Mathematics \\ Vol. 4, No. $3 \quad$ July, 1954}

Nelson Dunford, Spectral operators ........................ 321

John Wermer, Commuting spectral measures on Hilbert space.......... 355

Shizuo Kakutani, An example concerning uniform boundedness of spectral measures...................................... 363

William George Bade, Unbounded spectral operators .............. 373

William George Bade, Weak and strong limits of spectral operators ...... 393

Jacob T. Schwartz, Perturbations of spectral operators, and applications. I. Bounded perturbations ........................... 415

Mischa Cotlar, On a theorem of Beurling and Kaplansky............... 459

George E. Forsythe, Asymptotic lower bounds for the frequencies of certain polygonal membranes ............................... 467 